\title{
Recreo en casa. Aprender a convivir a través del juego
}

\section{Recess at home.} Learn how to live with others through play

\author{
VALERIA ACEVEDo* \\ JULIANA GUTIÉRREZ* \\ MARCIAL HunEEUS ${ }^{* * *}$ \\ MARGARITA PUGA ${ }^{* * * *}$
}

Este artículo sistematiza la experiencia de la propuesta educativa "Recreo en Casa", que busca el desarrollo socioemocional a través del juego de niños, niñas y adolescentes. Se trata de un programa de formación de acompañantes educativos (paradocentes, docentes y directivos) para adquirir herramientas concretas que les permitan construir una cultura de juego en la comunidad escolar y facilitar experiencias lúdicas en el contexto de educación a distancia producto de la COVID-19. Se recoge la experiencia de implementación del programa en 14 establecimientos educativos de Chile y presenta consideraciones teóricas sobre los beneficios del juego para la convivencia y el desarrollo socioemocional, físico y cognitivo de los estudiantes. Se problematiza, además, el rol de los acompañantes educativos para que, durante el recreo, sean actores clave en la construcción de la convivencia.

\section{Palabras clave:}

juego, desarrollo socioemocional, convivencia escolar, aprendizaje, recreo

Recibido: 19 de enero de 2021 | Aceptado para su publicación: 2 de septiembre de 2021 | Publicado: 26 de octubre de 2021

Cómo citar: Acevedo, V., Gutiérrez, J., Huneeus, M. y Puga, M. (2021). Recreo en casa. Aprender a convivir a través del juego. Sinéctica, Revista Electrónica de Educación, (57), e1273. https://doi. org/10.31391/S2007-7033(2021)0057-015 
This paper presents our experience implementing the educational program Recreo en Casa (Recess at home), which aims to impact on the socioemotional development of children and adolescents through play. This is a training program for school staff members who take part in supervising students' recess (teachers, directors, and other school staff members), to acquire tools and skills that will enable them to build a Play Culture in the school community and to create playful experiences in the context of the COVID-19 pandemic and long-distance education. This paper presents the experience of 14 schools in Chile, supplemented by theory on the benefits of play for students' wellbeing and their socioemotional, physical, and cognitive development. We also question the role of recess-staff members as key actors in the construction of a positive environment during recess time.

\section{Keywords:}

play, socio-emotional development, school wellbeing, learning, recess

\footnotetext{
* Ingeniera Civil de Industrias. Directora de Planificación y Desarrollo de Fundación Patio Vivo. Líneas de investigación: espacios con foco en infancia, mejoramiento escolar, ciencia del mejoramiento. Correo electrónico: valeria@patiovivo.cl

** Magíster en Psicología Educacional. Psicóloga de Educación y Convivencia de Fundación Patio Vivo. Líneas de investigación: asesoramiento psicoeducativo para la enseñanza y el aprendizaje de la lectura y la escritura. Correo electrónico: juliana@patiovivo.cl

${ }^{* * *}$ Magíster en Literaura. Director de Educación y Convivencia de Fundación Patio Vivo. Miembro de la Red Latinoamericana de Convivencia Escolar. Líneas de investigación: convivencia escolar, juego libre, desarrollo socioemocional, espacios con foco en infancia. Correo electrónico: marcial@patiovivo.cl

**** Psicóloga y diplomada en Pedagogía de la Convivencia y Desarrollo de Competencias Socioemocionales y Éticas. Profesional de Educación y Convivencia de Fundación Patio Vivo. Líneas de investigación: convivencia escolar, desarrollo socioemocional, psicología infanto-juvenil, espacios con foco en infancia. Correo electrónico: margarita@patiovivo.cl
} 


\section{INTRODUCCIÓN Y JUSTIFICACIÓN}

T a pandemia de la COVID-19 comenzó a propagarse a inicios de 2020 en Chile y en muchos países ocasionó el cierre presencial de los establecimientos educativos. Esto transformó de manera radical la rutina escolar e implicó un gran desafío para los equipos docentes, que debieron buscar nuevas estrategias pedagógicas y canales de comunicación para llegar a los estudiantes y sus familias.

Al momento de diagnosticar la realidad de los 14 colegios con los que se estaba trabajando, se evidenció una gran inestabilidad emocional en las familias y en los equipos educativos; además, el confinamiento redujo de modo drástico el tiempo de juego libre y activo en el exterior, así como exigió de un momento a otro que los equipos escolares comenzaran a comunicarse por medios digitales. Lamentablemente, al suspenderse las clases presenciales, y dada la petición de permanecer en casa, se acabaron los espacios de recreación y recreo escolar. Lo grave es que también se redujo el uso de plazas y parques, con lo cual niños, niñas y adolescentes (NNA) dejaron de jugar al aire libre con sus pares. A ello debemos agregar que muchos estudiantes viven en casas pequeñas o en condición de hacinamiento, lo que intensificó la sensación de encierro y redujo sus oportunidades de jugar con libertad y moverse en contacto con la naturaleza.

Recreo en Casa es un programa de formación socioemocional que Fundación Patio Vivo diseñó especialmente para acompañantes educativos a modo de respuesta a algunas necesidades y condiciones propias del contexto pandémico; es decir, para aportar a que los procesos de enseñanza y aprendizaje a distancia, mediante aplicaciones como Zoom o Meet, fueran mejores y para dar apoyo al bienestar integral de los diferentes miembros de la comunidad educativa; esto, considerando que hubo -y todavía hay- confinamientos bastante prolongados producto de la gravedad de la situación sanitaria en Chile.

El programa Recreo en Casa tiene como objetivo principal promover el desarrollo socioemocional de NNA y adultos a través del juego al valorar el tiempo de recreación. Por ello, el trabajo de formación socioemocional con acompañantes educativos tiene una doble intención: por un lado, llegar a impactar positivamente a NNA y, por otro, contribuir a la formación y experiencia de docentes y otras/os asistentes de la educación. De este modo, buscamos aportar y visibilizar la importancia del bienestar emocional en la convivencia escolar (Fierro y Carvajal, 2019), tanto de los estudiantes como de los mismos educadores, para la adquisición de aprendizajes.

\section{MARCO TEÓRICO}

\section{La importancia del juego}

A finales del siglo XIX y principios del XX, se comenzó a considerar el juego como una actividad en la que se producen diversos aprendizajes. Desde la pedagogía, Friedrich Fröebel, Rudolf Steiner, John Dewey, María Montessori y Loris Malaguzzi, entre otros, y, desde la psicología, Jean Piaget y Lev Vygotsky reconocieron en el juego un catalizador de aprendizajes físicos, socioemocionales y cognitivos. Huizinga (1972), en su libro Homo ludens, de 1938, planteó que el juego es más antiguo que la cultura, incluso que el juego es la actividad que conforma y desde la cual se desarrolla la 
cultura. Propuso que jugar caracteriza mejor al ser humano que el pensar y el hacer. Identifica el juego como una actividad libre y seria que permite explorar y conocer el mundo.

Huizinga caracteriza el juego como una actividad libre que se realiza por placer, como una experiencia siempre nueva, que implica inventar, ser creativos, flexibles y acodar significados comunes. "Es, en este sentido, imprescindible para la persona, como función biológica, y para la comunidad por el sentido que encierra, por su significado, por su valor expresivo y por las conexiones espirituales y sociales que crea" (Huizinga, 1972, p. 22).

El vínculo entre el juego y la cultura se observa, por ejemplo, en el juego simbólico en el cual niños y niñas representan y reviven las acciones de los adultos cercanos, juegan a cocinar, al doctor, a trabajar, manejar o a hablar por teléfono. Mediante sus juegos, conocen el mundo adulto. En el juego simbólico de los niños y las niñas es posible observar un espejo del mundo adulto: imitan nuestras acciones y las presentan sin un juicio. Así, van asimilando y comprendiendo características de los adultos/as que los cuidan y de la misma sociedad.

Desde la perspectiva sociocultural, el juego es entendido como una herramienta mediadora que contribuye al desarrollo de competencias cognitivas y comunicativas, ya que, cuando jugamos -tanto niños como adultos-, compartimos signos y construimos lenguaje en común (Vygotsky, 1979). En este sentido, Vygotsky (1993) plantea que el aprendizaje y conocimiento de la cultura se elabora y recrea en el proceso del juego infantil, al reproducir y asimilar activamente lo que observan en los adultos, y desarrollan el lenguaje, las actitudes y las habilidades primordiales que necesitarán para su actividad futura: "Poniendo en cada oportunidad al niño en situaciones siempre nuevas, sometiéndolo a condiciones también siempre nuevas, el juego lo obliga a diversificar ilimitadamente la coordinación social de sus movimientos y le enseña una flexibilidad, plasticidad y aptitud creativa como ningún otro ámbito de la educación" (Vygotsky, 1993, p. 162). El juego despierta la atención y el interés de NNA; por ello, Vygotsky lo vincula al aprendizaje, dado que se requiere el interés para aprender.

En la actualidad, existe un consenso sobre los beneficios del juego para el desarrollo físico, socioemocional y cognitivo de NNA. Milicic y López (2010) destacan el carácter social del juego para aprender a convivir, empatizar y a ser resilientes. Proponen que el juego es la principal instancia de aprendizaje antes de los seis años, ya que la vida social, el lenguaje, el equilibrio, la motricidad, entre múltiples capacidades, se adquieren jugando.

Para Fjortoft (2001), los NNA que juegan con regularidad en medios ambientes naturales muestran habilidades motoras más avanzadas, incluyendo la coordinación, el balance y la agilidad, y se enferman menos seguido. Asimismo, cuando juegan en medios ambientes naturales, su juego es más diverso, con imaginación y creatividad, lo que fomenta las habilidades del lenguaje y la colaboración.

En los programas pedagógicos nacionales (Chile) está muy presente el juego, especialmente en educación parvularia y en los años iniciales de educación básica o primaria. Las nuevas Bases Curriculares de Educación Parvularia (Mineduc, Subsecretaría de Educación Parvularia, 2018) lo incorporan como un eje fundamental 
para el aprendizaje, y es uno de los ocho principios pedagógicos. Sin embargo, en el quehacer diario de jardines infantiles y colegios es común que se privilegie la enseñanza de contenidos académicos por sobre las instancias de juego y juego libre.

\section{Disminución del juego libre y activo en el exterior}

Desde la investigación se plantea la disminución progresiva del tiempo de juego libre durante la infancia. Gray (2016) postula que, a partir de mediados del siglo XX, se ha producido un progresivo declive del juego al aire libre, que ha aumentado dramáticamente en los últimos veinte años, lo que está privando a niños y niñas de la oportunidad de fomentar habilidades socioemocionales que se adquieren de forma autónoma a través del juego. Plantea que los NNA necesitan jugar en libertad para desarrollarse: "Es el medio por el que los niños aprenden a hacer amigos, a vencer sus temores, a resolver sus propios problemas $\mathrm{y}$, en general, a controlar sus propias vidas. Es asimismo el medio básico por el que los niños practican y adquieren las capacidades físicas e intelectuales esenciales para el éxito en su cultura" (Gray, 2016, p. 22).

A través del juego libre, los niños y las niñas aprenden desde sí mismos, descubren lo que les gusta hacer y establecen amistades. Además, comprenden que las reglas, así como las normas sociales, son arbitrarias, responden a contextos históricos y, por tanto, modificables. Gray (2016) afirma que le preocupa que, dada la ausencia de experiencias de juego libre, se forme una generación que desconozca sus verdaderos intereses, carezca de autonomía y motivación para hacer su camino en la vida. Plantea que las habilidades que se adquieren con el juego libre con los pares no es posible que los adultos las enseñen. Advierte que la disminución del juego ya está mostrando un impacto negativo en el desarrollo físico, socioemocional y cognitivo de los NNA.

Gill (2007), de manera complementaria, señala que los NNA tienen pocas instancias de autonomía y libertad para jugar. Advierte que se han restringido sus posibilidades de moverse solos y de jugar de modo autónomo con otros NNA en el espacio público, dado que, por las características de la ciudad contemporánea, a menudo están bajo el control y la supervisión de los adultos. Además, por hacer más segura su experiencia, se ha limitado su campo de exploración y de juego con desafío, lo que tiene un impacto negativo en la adquisición de capacidades físicas, la autorregulación y de dirigir su propio aprendizaje. Destaca que, al jugar con desafío, los NNA aprenden a tomar decisiones, se ponen a prueba y adquieren habilidades socioemocionales.

Desde una perspectiva vinculada a la relación y el contacto con la naturaleza, Louv (2018) propone el concepto déficit de naturaleza para referirse a los NNA que crecen en grandes ciudades sin la posibilidad de interactuar y jugar al aire libre. Postula que el crecimiento urbano y el estilo de vida moderno han alejado a los NNA de la posibilidad de explorar e interactuar con sus pares de forma cotidiana en contacto con la naturaleza. Advierte que esta privación está teniendo un impacto negativo en el desarrollo físico, cognitivo y social de NNA. Hanscom (2016) también ha abordado la disminución del juego y el impacto negativo que está teniendo en ellos para la adquisición de habilidades motoras, la atención y la integración sensorial.

La disminución del juego en la infancia se condice con los resultados de la Encuesta Nacional de Primera Infancia (Junji/Unesco/Unicef, 2010), que da cuenta de los 
hábitos de crianza de las familias chilenas: el $43 \%$ de los padres y madres no juega con sus hijos más de una o dos veces por semana y el $40 \%$ no los lleva a espacios fuera del hogar a jugar con otros niños, mientras que casi el $60 \%$ no realiza juegos o actividad física fuera del hogar. Así, los mismos hábitos de crianza de las familias chilenas limitan las instancias de juego de NNA.

Esta problemática se está viendo incrementada con la pandemia, dado que se redujeron las posibilidades de desplazamiento, se cerraron las escuelas, las plazas y se han instalado largas cuarentenas. En contextos urbanos es común que muchos NNA no tengan la posibilidad de jugar con pares ni de estar al aire libre. Esta situación, prolongada durante más de un año, está teniendo un impacto negativo en la salud física y mental de NNA. El U-Report: El impacto del COVID-19 en la salud mental de adolescentes y jóvenes, del Fondo de las Naciones Unidas para la Infancia (Unicef, 2020), encuestó a más de ocho mil adolescentes y jóvenes de Latinoamérica y el Caribe, y reportó un aumento en la ansiedad (27\%) y en la depresión (15\%), en tanto que el $73 \%$ de los encuestados manifestó la necesidad de pedir ayuda en relación con su bienestar físico y mental. Asimismo, el $46 \%$ siente menos motivación para realizar actividades que normalmente disfrutaba. Por otra parte, el Mapa nutricional, de la Junta Nacional de Auxilio Escolar y Becas de Chile (Junaeb, 2020), identificó el impacto de la pandemia en el aumento de la obesidad, que llegó al 54.1\% entre sobrepeso y obesidad, mientras que solo el $38.2 \%$ se encuentra en el rango normal, lo que se vincula a la alimentación y la disminución de la actividad física.

\section{La importancia del recreo}

El juego cumple un rol central en la promoción de habilidades socioemocionales, físicas y cognitivas en NNA. Dada la baja cultura de juego de las familias, la inseguridad urbana y la jornada escolar completa, en un contexto normal, el recreo es la principal instancia de juego al aire libre que tienen NNA en la que adquieren y potencian sus habilidades. Sin embargo, si el recreo no se gestiona de modo adecuado, puede ser un momento conflictivo e incluso de agresiones. En la Encuesta Nacional de Violencia en el Ámbito Escolar (Ministerio del Interior y Seguridad Pública, 2014), el 33.6\% de los estudiantes declaró ser agredido con regularidad. Actualmente, hay violencia en las escuelas y el recreo es uno de los espacios donde ocurre.

Una estrategia para tratar los problemas de convivencia escolar consiste en abordarlos desde el juego en el tiempo de recreo. McNamara, Colley y Franklin (2015) destacan el valor del recreo y proponen cuatro variables: la cultura de recreo; la importancia de modelos de conducta saludables en el patio; la necesidad de una variedad de actividades y opciones durante el recreo; y la relevancia del espacio y la distribución espacial. Las tres primeras variables se vinculan a la capacitación y el trabajo con las comunidades educativas. Esto es en especial relevante dado que, como plantean McNamara, Colley y Franklin (2015), en el contexto canadiense, y en el chileno también, no hay demasiados criterios sobre los llamados inspectores, acompañantes de patio, tutores o acompañantes educativos. No se pide formación en educación ni hay un número definido de adultos por número de NNA. Sus funciones, en general, consisten en supervisar e intervenir en caso de incidentes o accidentes. 
La Academia Americana de Pediatría (2013) afirma que el tiempo de recreo es necesario para elaborar los aprendizajes académicos, dado que la mente necesita descansar para predisponerse a los nuevos desafíos. Este tiempo de uso libre entre una actividad y otra es igualmente importante para las diferentes edades, y es una instancia central del proceso formativo: "Recess serves as a necessary break from the rigors of concentrated, academic challenges in the classroom. But equally important is the fact that safe and well-supervised recess offers cognitive, social, emotional, and physical benefits" (p. 183).

La misma academia postula que las interacciones entre compañeros durante el recreo son un complemento único para los aprendizajes formales, en las cuales se adquieren habilidades de comunicación, negociación, cooperación, intercambio, solución de problemas y la resiliencia. Estas habilidades son la base para un desarrollo sano y un elemento fundamental de la experiencia escolar.

Un recreo bien gestionado puede entregar a los NNA un espacio valioso para la expresión de sus diferentes caracteres e intereses, y hacer posible el juego, el juego libre y la socialización. En un buen recreo se aprende a convivir, los estudiantes construyen amistades, fortalecen sus vínculos y se va generando apego escolar, lo cual predispone a un mejor aprendizaje en las diversas asignaturas. En ese sentido, el recreo no es solo un tiempo entre clases, sino una valiosa instancia de aprendizaje.

A diferencia de lo que ocurre en la sala de clases, donde el docente guía y decide qué se va a aprender (los contenidos de las distintas asignaturas), de qué forma (clase expositiva, trabajo individual o grupal) y con quién se va a interactuar (por lo general, niños y niñas del mismo curso y edad), en el recreo los estudiantes encuentran libertad para decidir qué quieren hacer, cómo y con quiénes. Esto es importante para aprender a convivir en una sociedad democrática y diversa. En el recreo pueden jugar, conversar y compartir con niños y niñas de otros cursos y, de esta manera, al socializar aprenden con y de sus pares, y también desarrollan competencias socioemocionales, como la empatía, la autonomía, la adaptación al cambio y la resiliencia. Así, los NNA descubren sus intereses, hacen amistades, colaboran grupalmente y toman decisiones.

\section{El rol de los adultos en el recreo}

Como plantean McNamara, Colley y Franklin (2015), la gestión del recreo requiere cuatro ámbitos, tres de los cuales se relacionan con el quehacer de los acompañantes educativos: la cultura de recreo, la importancia de modelos de conducta saludables en el patio, y la necesidad de una variedad de actividades y opciones durante el recreo. El programa Recreo en Casa eligió trabajar con estos actores clave en la rutina escolar para empoderarlos y transformar su función y quehacer en el recreo. Se buscó resignificar el rol tradicional de supervisores para adquirir un carácter más proactivo en el desarrollo y la promoción de una cultura de juego y buena convivencia.

También se privilegió que los acompañantes educativos contaran con herramientas lúdico/pedagógicas para enseñar juegos, mediar conflictos y contener emocionalmente a los estudiantes. No obstante, transformar modelos de conducta requiere autopercepción, conciencia y autocuidado. Siegel y Bryson (2015) sostienen que, para contener y empatizar con NNA, es indispensable que los adultos estén estables 
y tranquilos en términos emocionales; es decir, que reconozcan sus emociones y limitaciones antes de mediar o intervenir de modo directo con NNA.

Por otra parte, Cyrulnik (2019) indica que las figuras de apego secundario cumplen un rol fundamental en el fomento de la resiliencia. Define la resiliencia como la capacidad de iniciar un nuevo desarrollo después de un trauma o una situación de gran dificultad. Plantea que las personas resilientes, que logran empezar de nuevo tras una crisis o trauma, han establecido vínculos de apego secundario con familiares o adultos que conocían en sus escuelas, personas que creían en ellos y los apoyaban para que les fuese bien en la vida. En este sentido, los acompañantes educativos tienen la potencialidad de ser esas figuras de apego secundario, de apoyar y convertirse en actores de resiliencia, algo en especial valioso dada la pandemia.

Es indudable que el cierre de las escuelas producto de la pandemia presentó el desafío de replantear nuevas estrategias para seguir formando a NNA. Dichas estrategias, en la mayoría de los casos, se centraron en actividades académicas. Sin embargo, Recreo en Casa se presentó como un programa complemento para las escuelas a fin de propiciar y priorizar el desarrollo socioemocional a través del juego, además de ser un espacio de contención para los equipos educativos.

\section{OBJETIVOS}

El objetivo principal consiste en promover el desarrollo socioemocional de NNA y adultos a través del juego. Los objetivos específicos son: identificar el recreo como una instancia de aprendizaje; visibilizar la importancia del tiempo de juego y recreo; resignificar el rol de los acompañantes educativos como promotores del juego y modelos de conducta; crear un espacio de contención para el equipo de patio, convivencia y docentes; y prepararse para la vuelta a clases presenciales.

\section{METOdología}

Trabajamos con una metodología lúdica y práctica para propiciar la adquisición de herramientas concretas para el juego y la contención emocional. Diseñamos un programa de ocho talleres por colegio, en los que se realizan actividades desde la conciencia del cuerpo y la voz, y se establece una relación con la propia infancia. Los NNA están en etapas de crecimiento físico y necesitan moverse, sentir su respiración y su cuerpo. Propusimos empatizar con el momento vital de los estudiantes mediante juegos y actividades lúdicas para focalizar su atención en el cuerpo y el ritmo. Dada la importancia de la voz en el quehacer educativo, en especial en el contexto de clases y actividades sincrónicas, incorporamos actividades de autopercepción y autocuidado de la voz. El juego y el recreo en casa se abordaron como una instancia pedagógica que ayuda a contrarrestar la inestabilidad emocional propia del contexto pandémico y que, a su vez, promueve el desarrollo de habilidades como la resiliencia, la empatía y la creatividad, entre otras competencias socioemocionales.

Los talleres se llevaron a cabo en la totalidad de los casos de forma remota a través de la plataforma virtual Zoom. La metodología se basó en actividades prácticas y lúdicas orientadas a promover la reflexión, la creatividad y la co-construcción de 
conocimientos para el desarrollo de una cultura de juego en la comunidad. Esta metodología fue elaborada e implementada por el equipo multidisciplinario (educación, psicología, danza, voz y arquitectura) de Fundación Patio Vivo. Durante 2020 y 2021, y a lo largo de los ocho talleres, se abordó el quehacer educativo desde el trabajo integral: movimiento, uso de la voz y el ritmo, encuentro con otros desde el diálogo y la empatía, perder el miedo al ridículo y volver a conectar con la infancia; se entregaron herramientas concretas para poder jugar por medio de las pantallas y problematizando estrategias para la vuelta presencial. En algunos casos, se utilizaron presentaciones expositivas y en otros se organizó la sesión en torno a dinámicas artísticas y de expresión corporal (danza, canto y dibujo). En todos los casos hubo instancias de diálogo y reflexión entre participantes.

De esta forma, Recreo en Casa, además de ser un programa de formación socioemocional, fue puesto en práctica como una experiencia de autocuidado para acompañantes educativos, y también como un espacio para fortalecer la comunicación entre los miembros de la comunidad educativa. En este sentido, ha sido fundamental crear un espacio de contención, apoyo y reflexión sobre la experiencia compartida de la pandemia, siempre cuidando que las actividades fueran lúdicas, didácticas $\mathrm{y}$ acotadas a los tiempos disponibles.

\section{IMPLEMENTACIÓN}

Durante 2020 y 2021 se capacitó a más de 200 acompañantes educativos, docentes y directivos de 14 establecimientos educacionales de dependencia municipal y particular subvencionado ubicados en Chile. Cabe mencionar que la totalidad de estos presenta un alto índice de vulnerabilidad. Esto es relevante dado el contexto habitacional de los estudiantes, pues, en muchos casos, es de hacinamiento, comúnmente sin patio o con escasas instancias de acceso a parques y plazas seguras para jugar al aire libre. El programa de formación está compuesto por ocho talleres. En el primer semestre de 2020 se trabajó con siete establecimientos y, luego, en el segundo, se incorporaron los siete restantes. Se ofrecieron seis talleres en cada uno de los establecimientos del primer grupo y cuatro en los del segundo grupo. Durante 2021 se trabajó, junto con los 14 establecimientos, para brindar 112 talleres.

Las estrategias para promover el juego y el movimiento de NNA fueron tres: recreos en línea; juegos y actividades lúdicas en clases sincrónicas; y videos realizados por los mismos acompañantes de recreo para invitar a jugar. Para las dos primeras estrategias, se modelaron en los talleres formativos una gran variedad de actividades y opciones de juegos para llevar a cabo a distancia. Asimismo, para apoyar a las escuelas que comenzaron a volver a clases presenciales, se elaboraron los documentos "Baúl de juegos", para actividades sincrónicas, y "Volver a encontrarnos en la escuela", orientaciones para el recreo en el contexto de la COVID-19.

En específico, el taller uno consistió en una introducción a la importancia del juego y el desarrollo socioemocional; el dos se centró en la relevancia de la contención en tiempos de crisis; el tres presentó diversas estrategias lúdicas para implementar a través de la pantalla y se abordó su rol como promotores de resiliencia; el cuatro se centró en visibilizar la relación entre el juego y el aprendizaje, y reflexionar sobre las competencias adquiridas durante la pandemia; el cinco destacó la importancia 
del juego libre y cómo este promueve la empatía y la autorregulación; el seis promovió el trabajo en equipo y la colaboración como característica esencial para abordar desafíos; el siete consistió en un trabajo con la identidad de cada participante, la autopercepción de las fortalezas y el uso de la voz como herramienta para llegar a los estudiantes; el ocho fue un taller de cierre en el que se habló de la relevancia de conectar con el desarrollo socioemocional y con el juego, en especial en momentos de crisis como la pandemia. Hubo reflexión respecto a los beneficios que estos talleres brindaron en el ámbito individual (autocuidado) y en el profesional (comunicación hacia NNA y hacia otros miembros de la comunidad educativa).

Hay que destacar que los talleres contribuyeron a la resignificación de las vicisitudes propias de la pandemia, ya que ayudaron a ver esta educación a distancia como una oportunidad para la innovación pedagógica y la mejora educativa hacia un tema tan necesario como lo es el desarrollo de habilidades y competencias socioemocionales.

Junto con el programa de formación Recreo en Casa, se implementaron estrategias paralelas. Según Patterson y colaboradores (2007), para lograr generar cambios en la cultura, fomentar hábitos e instalar nuevas prácticas en una comunidad, se requiere abordar la temática desde distintos ámbitos que se complementan entre sí, ya que solo una o dos estrategias no lograrán la sostenibilidad de las prácticas en el tiempo. Por ello, Recreo en Casa consideró distintas vías para desarrollar la cultura de juego desde la formación docente y paradocente, la práctica con estudiantes, el trabajo con directivos, las comunicaciones y los documentos institucionales.

Específicamente, se pusieron en marcha talleres formativos dirigidos a los acompañantes de recreo, docentes y directivos; acompañamientos en la implementación del programa con estudiantes; reuniones estratégicas con el equipo directivo de cada escuela; estrategias comunicacionales e informativas dirigidas a toda la comunidad escolar; revisión y asesoría en la actualización del reglamento interno o manual de convivencia escolar de los establecimientos; y la entrega de materiales complementarios como cápsulas de juego y documentos orientadores para el recreo presencial.

\section{RESULTADOS}

\section{Resultado de estrategias para promover el juego}

Para que los acompañantes educativos pusieran en práctica con sus estudiantes lo aprendido en los talleres formativos, se elaboraron tres estrategias para llevar el juego y el recreo a las casas: videos que invitan al juego, recreos en línea y actividades lúdicas o juegos durante las clases sincrónicas. Los establecimientos educacionales aplicaron, de acuerdo con la pertinencia a su comunidad educativa, una, dos o las tres estrategias, lo cual permitió alcanzar a un total estimado de 3,660 estudiantes desde prebásica a enseñanza media.

\section{- Cápsulas de juego}

Los participantes de los talleres grabaron videos para enseñar juegos a sus estudiantes e invitarlos a jugar en casa junto con sus familias. En un comienzo, los videos fueron editados por el equipo de Patio Vivo y, paulatinamente, los equipos educativos comenzaron 
a elaborar sus propias cápsulas. En total, se realizaron 35 videos que fueron difundidos en las redes sociales de cada establecimiento y en el canal de YouTube de la Fundación Patio Vivo. Esta estrategia fue la más recurrente durante los primeros meses de la pandemia, dado que en los colegios había escaso manejo de las tecnologías, aún no se daban clases sincrónicas, y el video era una forma fácil y directa de llevar el juego a los niños y las niñas. Además, dos colegios propusieron a sus estudiantes que también hicieran videos que invitaran a jugar a sus compañeros/as a fin de involucrarlos en la construcción de una cultura de juego.

- Juegos y actividades lúdicas en clases sincrónicas

Algunos docentes y paradocentes que participaban en clases sincrónicas comenzaron a recurrir a juegos y actividades lúdicas durante las clases de las diferentes asignaturas. Los juegos se realizaron al comienzo, en medio o al finalizar la clase, lo cual tuvo un impacto en la asistencia y puntualidad de los estudiantes para conectarse a las clases, y servía como herramienta para activar la atención. Esta estrategia fue la más utilizada, ya que su ejecución dependía de cada participante y no del grupo completo. También, se incorporaron juegos y actividades lúdicas a las horas de orientación. Los docentes comentaron que el juego permitía activar el vínculo, dado que los estudiantes encendían sus cámaras para poder participar, lo agradecían y, luego, se daba una instancia de compartir.

\section{- Recreos en línea}

Una vez que hubo mayor dominio de la tecnología y las plataformas de videollamada (GoogleMeet), varios establecimientos comenzaron a incorporar recreos en línea dentro del horario de clases sincrónicas. En estos se hacían juegos, ejercicios para moverse, relajar el cuerpo y actividades lúdico-participativas. Cada equipo definió la frecuencia de los recreos, la duración y los estudiantes a quienes iban dirigidos. La duración, por lo general, variaba entre 15 y 25 minutos, la frecuencia era entre una y tres veces a la semana, y algunos recreos estaban orientados a cursos específicos, mientras que otros mezclaban estudiantes de distintas edades. En estas instancias se buscó impulsar el trabajo en duplas de paradocentes para guiar los recreos, ya que en el trabajo colaborativo encontraban mayor confianza y seguridad para guiar las actividades, perder el miedo al ridículo y lograr el involucramiento de los estudiantes. Profesionales del equipo de Patio Vivo participaron en la planificación de los primeros recreos y acompañaron en la ejecución, según el requerimiento de cada colegio.

Estas tres estrategias desarrolladas por los equipos educativos fueron complementadas con diarios murales digitales informativos y cuatro cápsulas de Recreo en Casa elaboradas por el equipo de Patio Vivo. Estas cápsulas invitan a generar tiempos y espacios de recreo y juego en familia, y destacan lo simple y beneficioso que es jugar para conectarse con otros, sentirse bien y promover habilidades socioemo-

cionales. Estas cápsulas y los diarios murales se difundieron en las redes sociales de los establecimientos educacionales. 


\section{Resultado de los talleres}

A modo general, como efecto de los ocho talleres, hubo un incremento en la capacidad de adaptación y flexibilidad de los equipos educativos para continuar con su labor formativa a distancia. Esto fue posible gracias a que el programa Recreo en Casa se fue desarrollando y nutriendo de la experiencia de trabajo con los establecimientos educacionales entre el primer semestre de 2020 y el primer semestre de 2021. En la medida que pasaba el tiempo, se fueron incorporando las necesidades que iban surgiendo por parte de cada comunidad educativa (y siempre en relación con las condiciones de confinamiento que se presentaban). Esto dio como resultado que la metodología y los contenidos tuvieran un eje central claro: promover el desarrollo socioemocional y la cultura del juego y, al mismo tiempo, que estuvieran altamente vinculados a la experiencia y el contexto de las escuelas; es decir, la implementación fue basada en la escucha de las necesidades de cada comunidad, lo cual fue destacado por los participantes en la evaluación del programa.

También se destacó el carácter lúdico, reflexivo y contenedor de los talleres en un contexto muy exigente y desgastante: "A través de este taller tuvimos la oportunidad de jugar, reírnos, bailar y tener un espacio de relajación en medio de todo lo que puede significar el adaptarnos a esta nueva normalidad. Además, podremos realizar esto [actividades y juegos] cuando volvamos a nuestra escuela" (colegio 7). Se produjo una valoración del juego y de haber adquirido herramientas concretas para promoverlo con sus estudiantes.

La posibilidad de reflexionar sobre su experiencia de la pandemia y adquirir herramientas fue en particular significativo para el primer grupo, ya que, durante los primeros meses, entre mayo y junio de 2020, no había certezas acerca del retorno presencial a la escuela. La incertidumbre era parte de la realidad de los primeros meses de la pandemia. Los equipos educativos habían manifestado que no se sentían preparados ni con herramientas concretas para comunicarse de manera adecuada con los estudiantes y sus familias. Por esta razón, los primeros talleres tuvieron un enfoque exploratorio que permitió profundizar en el diagnóstico de las necesidades y hacer un acompañamiento cercano a los equipos educativos durante la etapa de adaptación a la metodología remota de los procesos. Incluso se asesoró a los equipos directivos en torno al uso de redes sociales y herramientas digitales.

En los talleres se recogieron las emociones experimentadas durante la pandemia, y predominaron las siguientes: incertidumbre, ansiedad, angustia, miedo, estrés, tristeza, entre otros. Tras realizar diversas actividades lúdicas desde el cuerpo y el movimiento, mencionaron que sintieron emociones como alegría, relajo, diversión, felicidad, entre otras. De esta forma, se contrastaron las emociones vivenciadas durante la pandemia en general con las experimentadas inmediatamente después de jugar (ver figura). Esta comparación para los equipos educativos evidenció el carácter sanador y liberador del juego, y la importancia de vincularse con otros. Además, permitió reflexionar sobre la importancia del autocuidado y abordar el rol de los acompañantes educativos como promotores del juego. En este sentido, para la contención y el bienestar emocional de sus estudiantes, en especial en el contexto de pandemia, era fundamental que los equipos de adultos que conformaban la comunidad educativa tuvieran instancias de autocuidado y apoyo mutuo. 


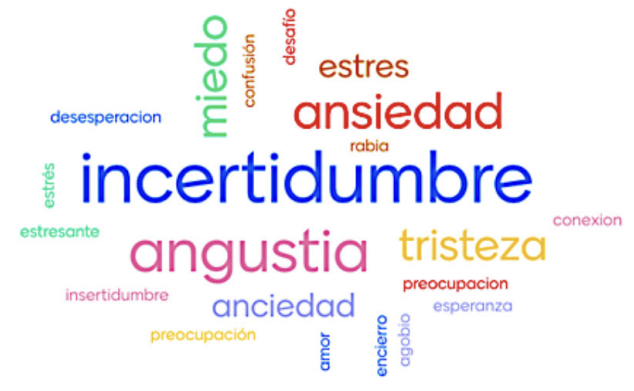

Emociones en la pandemia

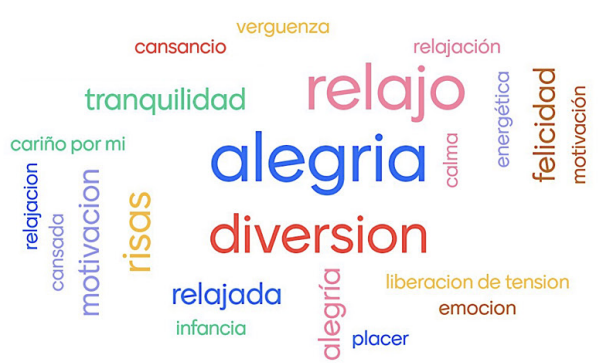

Emociones al jugar

Figura. Palabras escritas por los participantes en respuesta a las preguntas “¿qué emociones han surgido a partir de la pandemia?” y “¿qué sentiste al hacer los juegos?”, respectivamente, a través de la herramienta digital Mentimeter.

Sobre los talleres, se valoró un impacto positivo en distintos ámbitos del quehacer educativo:

Hay un cambio muy bueno en la escuela desde que partimos con Patio Vivo. Desde el juego de los niños, ahora tienen más conciencia de respetarse y compartir. Nosotras hemos podido conocernos más como equipo y desde otro lugar. Hemos podido generar cosas nuevas en la escuela y ha sido muy bueno para los profes y los niños. Me ha ayudado a poder conocerme a mí misma y moverme más. Estoy muy contenta del apoyo que nos dieron. Ha sido un crecimiento como colegio. Estoy profundamente agradecida (colegio 2).

Se destaca como una experiencia formativa entretenida y dinámica que les permitió mirar la educación desde otro lugar.

También se apreció el espacio de autocuidado y la posibilidad de aprender desde el cuerpo y los sentidos: "Solo tengo agradecimientos. Este ha sido un espacio de autocuidado, muy necesario. Hemos aprendido nuevas herramientas para llevarlo a la práctica. Necesitaba este espacio, donde podemos reflexionar con todo el cuerpo y aprender a través del juego" (colegio 4). Por otra parte, se destaca la importancia de empatizar con las necesidades de NNA a partir de las experiencias de juego: "Los talleres han sido un oasis en la pandemia. Una pasa a ser estudiante y a descubrir. Siento que construimos juntos este espacio. Nos dimos cuenta de que había una comunidad de niños esperando por nosotras. Estoy profundamente agradecida. Ahora soy más flexible, más abierta, más empática" (colegio 11). Se observó un cambio interno en las participantes para llevar las herramientas adquiridas a la formación de los estudiantes.

Finalmente, se mencionó la importancia de abordar el juego como una estrategia de aprendizaje y para mejorar la convivencia escolar:

Es una experiencia revolucionaria entender el juego realmente como una estrategia de aprendizaje. Reflexionar sobre el juego para sacar lo mejor de los niños, esa libertad que tiene el juego. No es algo aparte, el juego es fundamental para el aprendizaje y la sana convivencia. Recreo en casa es muy revolucionario, ha dado resultado y lo estamos llevando a los otros colegios de la red, para llegar a más y más niños. 


\section{EVALUACión DEL PROGRAMA}

Con la intención de recibir retroalimentación y continuar mejorando el programa, aplicamos una encuesta de evaluación de los talleres formativos y las estrategias de Recreo en Casa que enviamos a los participantes al finalizar el último taller. Los talleres fueron evaluados en las dimensiones metodología aplicada (ver gráfica 1), pertinencia del contenido de los talleres en el contexto de la pandemia (ver gráfica 2) y claridad en la entrega de la información por parte de los talleristas y la cercanía en el trato durante las sesiones (ver gráficas 3 y 4).

Gráfica 1. Evaluación de la metodología de los talleres

En una escala de 1 a 4, ¿cómo evaluarías la metodología de los talleres?

118 respuestas

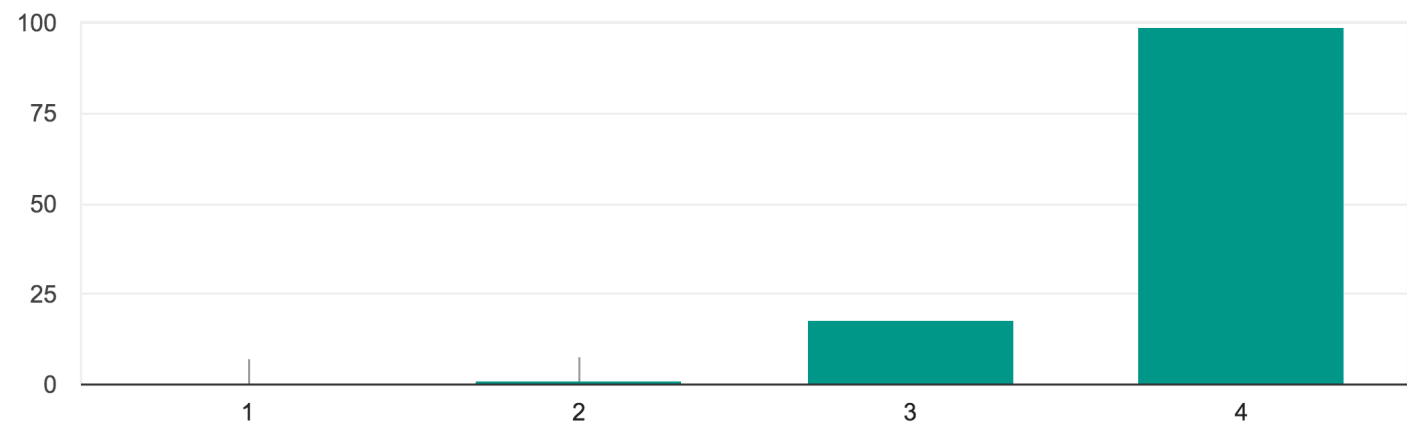

Gráfica 2. Evaluación de la pertinencia de los contenidos de los talleres

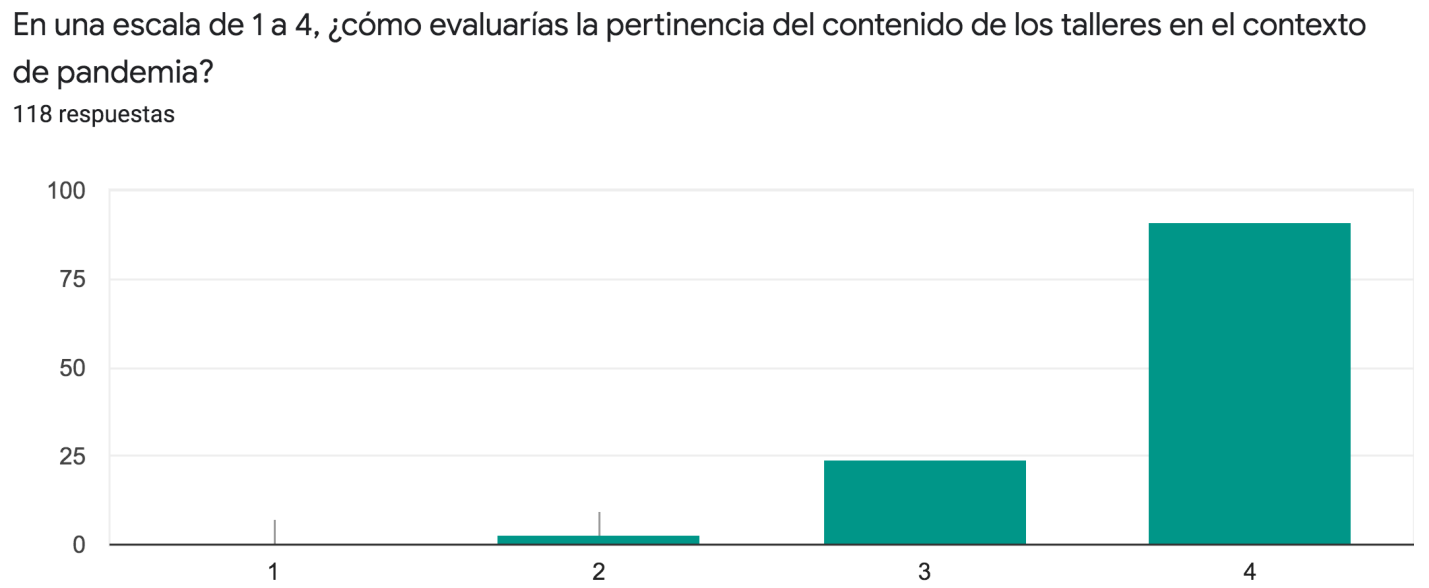




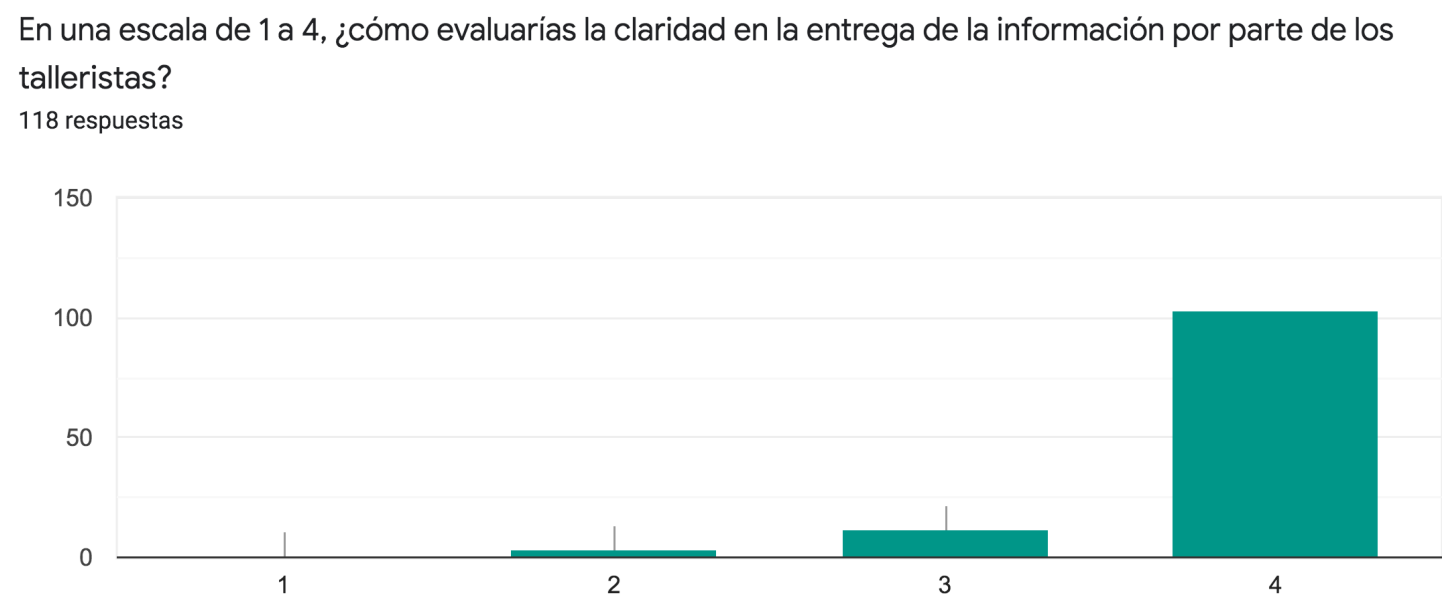

Gráfica 4. Evaluación que tuvo la cercanía en el trato por parte de los talleristas hacia los participantes de los talleres

En una escala de 1 a 4, ¿cómo evaluarías la cercanía en el trato de los talleristas durante las sesiones?

118 respuestas

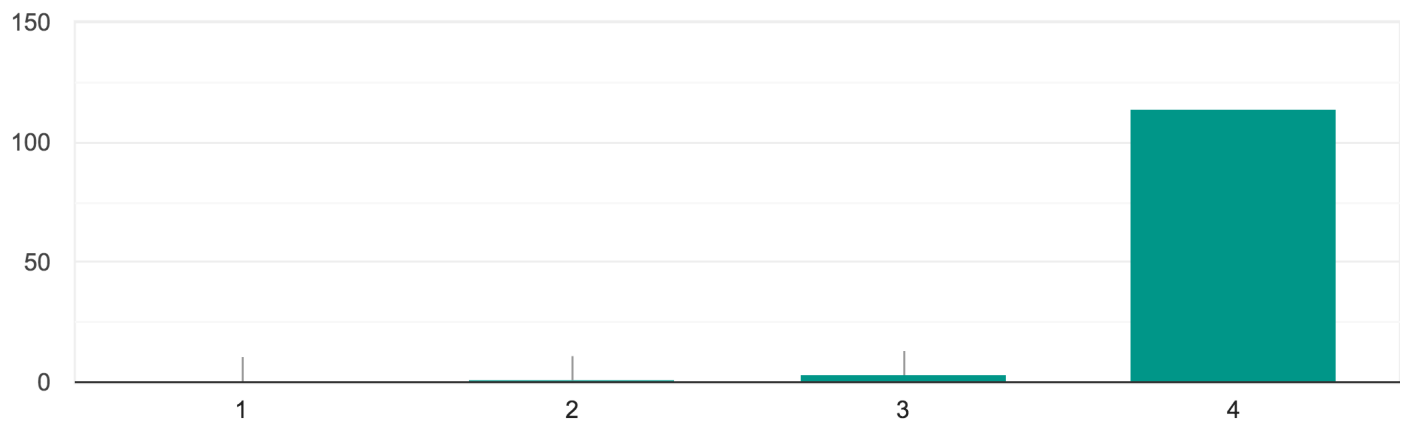

Los talleres han sido evaluados como "muy buenos", "muy pertinentes", "muy claros" y "muy cercanos" por más del 77\% de los participantes, lo que es un resultado muy satisfactorio. En cuanto a su utilidad, la encuesta proponía un espacio abierto para escribir lo que cada participante calificó como un aporte para su formación. Al preguntarles por la confianza ante un posible retorno a clases y a recreos presenciales, un $88.1 \%$ de los participantes consideró que cuenta con las herramientas necesarias para seguir fortaleciendo esta cultura de juego al momento del retorno presencial a clases (ver gráfica 5). 
Gráfica 5. Porcentaje de participantes que consideran que los talleres les han permitido adquirir más herramientas para seguir construyendo una cultura de juego cuando se vuelva a clases presenciales

¿Te sientes con herramientas para seguir construyendo una cultura de juego cuando se vuelva a clases presenciales?

118 respuestas

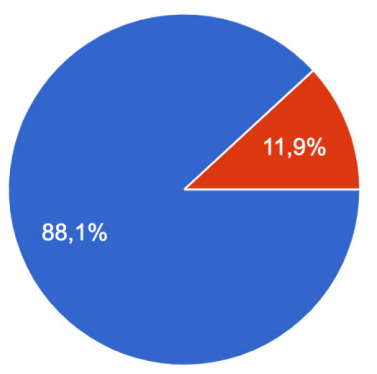

Si

Para medir la experiencia y satisfacción global de los participantes, utilizamos como indicador el Net Promoter Score (NPS), debido a la posibilidad de establecer un benchmark con otros programas. La distribución final mostrada en la gráfica 6 resulta en un NPS final del 78.9\%, al obtener resultados destacados, y nos pone el desafío de mejorar para las respuestas en los niveles inferiores.

Gráfica 6. Indicador NPS para medir la experiencia y satisfacción global de los participantes respecto al programa Recreo en Casa

En una escala de 1 a 10, ¿qué tan probable es que recomiendes estos talleres a equipos de otros colegios?

118 respuestas

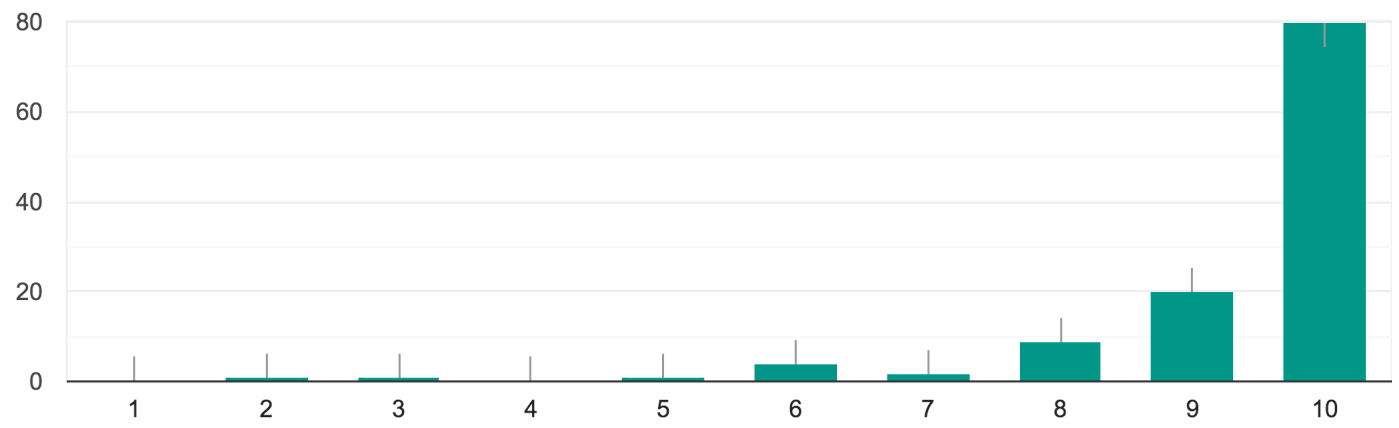

En último término, la gráfica 7 muestra que el $96.6 \%$ de los participantes en la formación declaró haber adquirido herramientas para promover el desarrollo socioemocional a través del juego. 
Gráfica 7. Porcentaje de participantes que consideran que los talleres les han permitido adquirir más herramientas para promover el desarrollo socioemocional a través del juego

¿Los talleres te han permitido adquirir más herramientas para promover el desarrollo socioemocional a través del juego?

118 respuestas

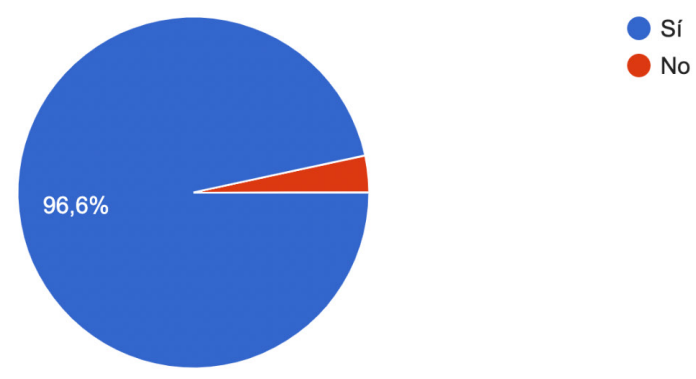

A modo general, observamos que los participantes compartieron a lo largo de los talleres diversos tipos de aprendizajes: digitales (uso de plataformas y herramientas para el trabajo remoto); socioemocionales; vinculares (la importancia del fortalecimiento de la relación familia-escuela); educativos (valoración de la cultura de juego en la comunidad y del trabajo en equipo). Esta valoración del trabajo en equipo y el deseo de desarrollar una cultura de juego y sana convivencia en la escuela se evidencia en los comentarios finales de los participantes.

Al cierre de los talleres, se destacó la adquisición de diversos aprendizajes vinculados a herramientas concretas para hacer juego por medio de la pantalla; mayor seguridad para realizar y enseñar juegos; valoración del espacio de autocuidado para apoyar a otros; la importancia del juego en la construcción de la convivencia escolar; entender el juego como un factor para el desarrollo integral de los estudiantes; y su relevancia en la formación de NNA.

En relación con las herramientas para implementar juegos, tanto en las clases como en el recreo, mencionan:

Ideas concretas para implementar en mis clases de Orientación, canciones, actividades de relajación, dinámicas de integración, entre otros (colegio 4).

Ahora conocemos diferentes juegos que, sin tener que usar algún tipo de material, pueden involucrar a los niños a dinámicas variadas y entretenidas (colegio 7).

Me motiva el buscar instancias dentro de la clase para favorecer el movimiento y el juego, entregando ideas que pueden ser fácilmente aplicadas y demostrando de forma práctica lo beneficioso que es (colegio 8).

De esta manera, se evidencia lo simple que es jugar, dado que no se requieren grandes elementos, sino disposición y motivación. Se mencionó que adquirieron mayor seguridad personal para liderar actividades lúdicas: 
La verdad me sentía incapacitada de hacer dinámicas vía online; el taller me abrió los ojos y me dio las herramientas para hacerlo (colegio 5).

Este espacio me ha permitido perder el miedo a la vergüenza y sentirme parte de un equipo de trabajo, logrando objetivos juntos (colegio 7).

Todo lo que nos proponemos, lo podemos hacer con nuestros estudiantes, aunque estemos a distancia y a través del juego podemos entregarles momentos significativos (colegio 11).

Yo creo que soy capaz de realizar distintos tipos de juegos y llevarlos a la realidad [...], me dio más personalidad porque, aunque ustedes no lo crean, soy muy tímida (colegio 7).

De a poco me empecé a enamorar del proyecto. Se van a extrañar las capacitaciones porque uno fluye en otra área distinta a lo que es tu cargo y eso te nutre. Vas aprendiendo a no solo aplicarlo en el colegio, sino que también en la vida y con niños de tu familia (colegio 1).

Como inspectora ha sido un aprendizaje notable: haber hecho videos, verme en esto de las pantallas, hacer juegos; jamás en mi vida me lo hubiese imaginado a la edad que tengo. No sentir el ridículo, porque uno se siente ridícula a cierta edad, pero al final uno pierde el pudor y lo logra. Frente a los niños, que son nuestros jueces, hemos tenido una muy bonita llegada y eso obviamente se agradece (colegio 13).

Creo que después de todos estos talleres, soy una persona más flexible, más abierta a cosas nuevas y más empática también (colegio 4).

Así, reconocen aprendizajes a nivel personal que les permiten realizar de mejor forma su labor pedagógica.

Se destacó también el espacio de autocuidado y la relevancia que tiene para cuidar y apoyar a los estudiantes:

En estos talleres hemos aprendido a darnos un tiempo para nosotras mismas y así estar preparados para poder contener y ayudar a los niños en el desarrollo de habilidades (colegio 12).

Para mí este fue un espacio de autocuidado muy necesario. Para aprender herramientas, reflexionar, mirar desde otro lugar y llevarlo a la práctica en el quehacer cotidiano, con los niños y niñas y profesores. Necesitaba ese espacio, a veces pasa mucho en el colegio, que los espacios para reflexionar son acotados, no lo podemos hacer como acá, que es con todo el cuerpo, reflexionar y sentir (colegio 9).

Se plantea la importancia de contar con más instancias de relajación, reflexión y juego a fin de contar con más herramientas para contener a los estudiantes. Asimismo, se reconoce la forma en que el juego aporta a la construcción de la convivencia y los vínculos dentro de la comunidad educativa: "Mi mayor aprendizaje hasta ahora en los talleres ha sido la importancia del juego y el movimiento para favorecer una mejor convivencia, motivar a la reflexión y desarrollar más cercanía con la comunidad escolar" (colegio 8).

También se menciona el lugar del juego en el desarrollo socioemocional de NNA:

Creo que al aprender a través del juego podemos llegar de mejor manera a los niños y podemos darnos cuenta de lo que siente cada uno de ellos, cómo están anímicamente y así poder luego intervenir (colegio 3) [y se valoran las oportunidades para socializar]: 
El juego es un medio que permite socializar, ya que se entra en contacto con otros niños, niñas y con adultos, aprendiendo a respetar normas de convivencia y a conocer el mundo que lo rodea (colegio 10).

Fue un descubrimiento, construimos juntos. Nos invitaron a construir todos juntos, todo lo que hemos ido haciendo en los talleres y lo traspasamos a los niños. Descubrir que hay una comunidad de niños y apoderados esperando participar de las actividades y hacer videos, fue gracias a estos talleres. Nos cuidamos nosotros, para cuidar mejor a los otros. Y nos dimos el permiso para descubrir cosas que no sabíamos que existían. Profundamente agradecida de todos (colegio 6).

Existe el reconocimiento de que las familias y los estudiantes también valoran lo que se ha implementado. Además, se destaca el desarrollo integral por medio del juego:

A través del juego podemos activar diferentes áreas de nuestro desarrollo (cognitivo, sensorial, emocional), además de las habilidades blandas (colegio 2).

Mediante el juego es posible generar aprendizajes significativos de manera integral [...] con cosas simples y sencillas podemos generar instancias de participación, contención, exploración que nos lleven a desarrollarnos como comunidad (colegio 11).

El juego abre espacios de colaboración, diálogo, cercanía y promueve el respeto al ser bien guiado. Es importante ser flexibles y tener una buena disposición al cambio. No hay que temer al jugar, ni sentir vergüenza porque sin importar la edad, cuando jugamos todos somos niños y niñas (colegio 14).

Se menciona la conexión con la propia infancia para así empatizar y llegar de mejor forma a NNA.

Finalmente, se valoró que la construcción de una cultura de juego tiene beneficios sistémicos en el proceso educativo:

Estos talleres nos ayudaron a reforzar un compromiso con una educación más consciente, más democrática y respetuosa de la infancia. El juego es un promotor transversal del "buen vivir" (colegio 1).

Yo antes pensaba en el colegio como un espacio de formación académica y ahora pienso en el colegio como un espacio de formación humana, de crecimiento personal y social en donde el juego forma parte fundamental de este importante proceso (colegio 12).

Aprendimos a desmitificar el juego, entenderlo y posicionarlo como una experiencia didáctica y de aprendizaje. El juego no es algo externo al conocimiento. Se puede potenciar y sacar lo mejor de los niños estimulando ese lado salvaje y libre, aprendiendo y promoviendo una sana convivencia escolar. Sin eso, no existe nada de lo que el colegio se propone. Recreo en Casa es una idea muy revolucionaria (colegio 7).

Estas evaluaciones dan cuenta del proceso de aprendizaje y la adquisición de herramientas para implementar con sus estudiantes.

\section{CONCLUSIONES Y REFLEXIONES FINALES}

Ante la extensión de los confinamientos, junto con el cierre físico de las escuelas producto de la pandemia, el objetivo de promover el desarrollo socioemocional a través del juego implicó un gran desafío. En el contexto normal, en un recreo bien gestionado, NNA tienen la oportunidad de jugar en el patio escolar todos los días de 
la semana; cuentan con un tiempo y un espacio determinado para ello. Sin embargo, durante la pandemia, la comunidad educativa dejó de tener contacto presencial y se vio fuertemente estresada ante la realidad. La experiencia cotidiana estaba atravesada por emociones como la incertidumbre, el miedo y la ansiedad, y se vieron afectados estudiantes, profesores, directivos y familias. Existía una preocupación por cómo continuar enseñando, aprendiendo y conviviendo en esas condiciones.

El programa Recreo en Casa pretendía ser un aporte en este momento tan crucial al complementar los esfuerzos que se estaban haciendo en los colegios con formación en desarrollo socioemocional a través del juego. El juego y el tiempo de recreación de NNA no podía descuidarse; al contrario, debía promoverse dado que las restricciones sanitarias lo estaban limitando. El trabajo de formación socioemocional con acompañantes educativos tuvo una doble intención: por un lado, llegar a impactar positivamente a NNA y, por otro, contribuir a la formación, contención y autocuidado de docentes y otras/os asistentes de la educación.

Con base en lo observado en los resultados y los testimonios, es posible afirmar que se cumplió el objetivo. Sus comentarios dan cuenta de una toma de conciencia respecto de su quehacer, en el cual el juego adquiere relevancia y, además, declaran sentirse más seguros para hacer actividades lúdicas con los estudiantes. Los acompañantes educativos tomaron conciencia del lado positivo que ha tenido el trabajo remoto, al reconocer que tanto ellos como los estudiantes tienen la capacidad de continuar aprendiendo cosas nuevas y, así, poder adaptarse a los nuevos desafíos. Además, se vio la importancia del recreo como instancia de aprendizaje y desarrollo socioemocional, y fue una de las actividades que los NNA más extrañaban de la presencialidad.

Los talleres abrieron la oportunidad de mirar la educación desde un lugar diferente; el mismo hecho de dejar de trabajar presencialmente en el establecimiento permitió que todos pudieran tomar perspectiva sobre el quehacer diario. Se adquirió conciencia de la relevancia del juego como catalizador de emociones positivas, que contribuyen a sobrellevar la incertidumbre, fortalecer los vínculos entre adultos y estudiantes, y fomentar habilidades y competencias socioemocionales como la empatía, la resiliencia, la creatividad, la autopercepción, entre otras. Se trabajó para resignificar el rol de los acompañantes educativos y pasar de un rol de supervisión a una función proactiva en la construcción de una cultura de juego para el desarrollo socioemocional.

Sin embargo, aún es necesario seguir fortaleciendo este objetivo. Los participantes se sienten con más herramientas y con la capacidad de llevarlo al recreo presencial, pero hay que ver su sostenibilidad en el día a día. De igual modo, las mismas restricciones sanitarias limitan las interacciones normales entre estudiantes y agregan tareas adicionales a los acompañantes, como cuidar el distanciamiento físico. Para apoyar en el retorno presencial, se compartió el documento Orientaciones para el recreo en contexto de la COVID-19, en el que, además de recomendaciones vinculadas a la gestión del ámbito sanitario, se compartieron distintos tipos de juegos con distanciamiento físico para enseñar en el recreo.

Luego de revisar los resultados y los testimonios, es posible concluir que Recreo en Casa, aparte de ser un programa de formación socioemocional, también fue una experiencia de autocuidado y fortalecimiento de los equipos educativos. Abordar el 
juego desde actividades que incorporan el movimiento corporal, la comunicación emocional, así como la expresión artística mediante una plataforma virtual y por videollamada era una metodología nueva.

Pese a las dificultades técnicas que se presentaron, la respuesta fue positiva: es posible construir una cultura de juego por canales digitales. Si bien muchos acompañantes educativos no estaban familiarizados con el uso de plataformas digitales, asumieron el desafío y aprendieron a encabezar actividades lúdicas en ese formato. Esto nos lleva a reflexionar que hoy, en medio de una pandemia y en un mundo cambiante, promover la cultura de juego y el desarrollo socioemocional permite a las comunidades educativas crear una instancia de libre aprendizaje en que NNA aprenden a socializar, resolver conflictos y ser resilientes.

Es necesario institucionalizar en documentos de gestión, como el manual de convivencia, el objetivo de promover una cultura de juego en el recreo, de modo que se haga parte de sus pilares y su misión. Para ello, hemos revisado los manuales de convivencia y estamos elaborando un documento que presente los beneficios de una cultura de juego para el desarrollo socioemocional y la importancia de resignificar el rol de los acompañantes educativos hacia un quehacer proactivo en la construcción de dicha cultura y su incorporación en los documentos institucionales. También, estamos trabajando en el desafío de crear la Red de Colegios Patio Vivo, en la que pretendemos generar espacios de comunicación entre los 14 colegios que participaron en los talleres para luego sistematizar cuáles son los puntos comunes entre todos y, así, proyectar que el programa se expanda a otras regiones de Chile y Latinoamérica.

\section{REFERENCIAS BIBLIOGRÁFICAS}

American Academy of Pediatrics (2013). The crucial role of recess in school. Pediatrics, vol. 131, núm. 1, p. 183. https://doi.org/10.1542/peds.2012-2993

Cyrulnik, B. (2019). Escribí soles de noche: literatura y resiliencia. Barcelona: Gedisa.

Fierro, C. y Carvajal, P. (2019). Convivencia escolar: una revisión del concepto. Revista Psicoperspectivas, vol. 18, núm. 1, pp. 1-19.

Fjortoft, I. (2001). The natural environment as a playground for children: The impact of outdoor play activities in pre-primary school Children. Early Childhood Education Journal, vol. 29, núm. 2. https://doi. org/10.1023/A:1012576913074

Gill, T. (2007). No fear, growing up in a risk averse society. Londres: Calouste Gulbenkian Foundation.

Gray, P. (2016). Libres para aprender, por qué el aprendizaje lúdico garantiza niños felices y seguros de sí mismos. México: Paidós.

Hanscom, A. (2016). Balanced and barefoot. Estados Unidos: New Harbinger Publications, Inc.

Huizinga, J. (1972). Homo ludens. Madrid: Alianza Editorial.

Junaeb (2020). Mapa nutricional. Santiago de Chile.

Junji/Unesco/Unicef (2010). Encuesta Nacional de Primera Infancia (pp. 4853). Santiago de Chile. 
Louv, R. (2018). Los últimos niños en el bosque. Madrid: Capitan Swing.

McNamara, L., Colley, P. \& Franklin, N. (2015). School recess, social connectedness, and health: a Canadian perspective. Health Promotion International.

Milicic, N. y López de Lérida, S. (2010). Hijos con autoestima positiva. Santiago, Chile: Editorial Paidós.

Mineduc, Subsecretaría de Educación Parvularia (2018). Bases curriculares Educación Parvularia. Santiago de Chile.

Ministerio del Interior y Seguridad Pública (2014). Encuesta Nacional de Violencia en el Ámbito Escolar. Santiago de Chile.

Patterson, K., Grenny, J., Maxfield, D., McMillan, R. \& Switzler, A. (2007). Influencer. The power to change anything. McGraw-Hill. https://doi. org/10.1036/007148499X

Siegel, D. J. y Payne Bryson, T. (2015). Disciplina sin lágrimas. Una guía imprescindible para orientar y alimentar el desarrollo mental de tu hijo. Barcelona, España: Ediciones B.

Unicef (2020). U-Report: el impacto del COVID-19 en la salud mental de adolescentes y jóvenes.

Vygotsky, L. (1993). Obras escogidas. Madrid: Visor.

Vygotsky, L. S. (1979). El desarrollo de los procesos psicológicos superiores. Buenos Aires: Grijalbo. 